\title{
Non-heat-treated frozen raspberries the most likely vehicle of a norovirus outbreak in Oslo, Norway, November 2013
}

\author{
M. EINÖDER-MORENO ${ }^{1,2}$, H. LANGE ${ }^{1,2}$, M. GREPP ${ }^{3}$, E. OSBORG $^{4}$, \\ K. VAINIO ${ }^{5}$ AND L. VOLD ${ }^{1}$ \\ ${ }^{1}$ Department of Infectious Disease Epidemiology, Norwegian Institute of Public Health, Oslo, Norway \\ ${ }^{2}$ European Programme for Intervention Epidemiology Training (EPIET), European Centre for Disease \\ Prevention and Control (ECDC), Stockholm, Sweden \\ ${ }^{3}$ Department of Public Health, Health Agency, Municipality of Oslo, Oslo, Norway \\ ${ }^{4}$ Norwegian Food Safety Authority, Oslo, Norway \\ ${ }^{5}$ Department of Virology, Norwegian Institute of Public Health, Oslo, Norway
}

Received 2 March 2015; Final revision 11 December 2015; Accepted 19 January 2016; first published online 16 February 2016

\section{SUMMARY}

In November 2013, the Norwegian Institute of Public Health was notified of a gastroenteritis outbreak following two meetings held at a conference centre. Identical food and beverages were served during the meetings. We investigated in order to identify the vehicle of infection and implement control measures. Meeting participants completed an online questionnaire on consumption of foods and beverages. We asked symptomatic participants to provide a stool sample. We defined a case as diarrhoea and/or vomiting in a participant who became ill within 3 days after the meeting. We calculated attack rates (AR) and adjusted risk ratios (aRR) with 95\% confidence intervals $(\mathrm{CI})$ using binomial regression. We conducted environmental investigations. Overall, 147/168 (88\%) participants responded, of which 74 (50\%) met the case definition. All five stool samples provided were norovirus positive. No kitchen staff reported being sick. Risk of illness was higher in those who consumed raspberry mousse (aRR 3.4, 95\% CI 1.4-8.2) and sliced fresh fruit (aRR $1 \cdot 9,95 \%$ CI 1·3-2.8). Seventy cases (95\%) ate raspberry mousse. Frozen raspberries used for the mousse were imported and not heat-treated before consumption. Non-heat-treated frozen raspberries were the most likely outbreak vehicle. Contamination by a food handler could not be excluded. We recommend heat-treatment of imported frozen berries before consumption.

Key words: Berries, Caliciviridae infections, disease outbreaks, foodborne diseases, norovirus.

\section{INTRODUCTION}

Norovirus is a RNA virus of the family Caliciviridae that frequently causes gastroenteritis in humans [1]. Norovirus are divided into five genogroups (GIGV), but only GI, GII and GIV are known to infect

\footnotetext{
* Author for correspondence: Dr M. Einöder-Moreno, Norwegian Institute of Public Health, Department of Infectious Disease Epidemiology, Postboks 4404 Nydalen, NO-0403 Oslo, Norway. (Email: Margot.Einoder.Moreno@fhi.no)
}

humans $[1,2]$. Norovirus is highly contagious and the infectious dose may be as low as 10-100 viral particles $[3,4]$. It is one of the most common causal agents of non-bacterial gastroenteritis outbreaks worldwide [3, 5]. The main transmission route is faecal-oral, and norovirus can spread via person-to-person contact, droplets, fomites (contaminated with vomitus or faecal matter) and the consumption of contaminated food or water $[1,3]$. The incubation period usually ranges between $12 \mathrm{~h}$ and $48 \mathrm{~h}$. Symptoms last on average 1-3 days and the most common are vomiting and/or 
watery diarrhoea, although nausea, abdominal pain, fever and headache can also be seen [6].

Norovirus outbreaks occur most often in the winter season. Foodborne outbreaks are frequently notified in large institutions and residential facilities such as healthcare institutions and daycare centres, cruise ships, military camps and hotels [3]. In the last 10 years norovirus has been the most frequently suspected agent in foodborne gastroenteritis outbreaks notified to the Norwegian Institute of Public Health (NIPH). In order to prevent outbreaks in healthcare settings, NIPH recommends that healthcare workers (HCWs) with symptoms of norovirus infection should wait $48 \mathrm{~h}$ after termination of diarrhoea and vomiting before returning to work [7].

On 11 November 2013, the Municipal Health Officer (MHO) of Oslo reported to the NIPH an outbreak of gastroenteritis among participants attending two different 1-day meetings organized at a conference centre in Oslo, on 4 November. The two meetings were held in different rooms, but all the participants were served the same selection of foods and beverages. Considering the time of onset and symptoms reported by the first reported cases, the MHO suspected norovirus as the causative agent. A multidisciplinary outbreak investigation team was established the day of the outbreak notification with partners from the NIPH, the Norwegian Food Safety Authority (NFSA) and the Municipal Health Services of Oslo. The objective of the outbreak investigation was to describe the extent of the outbreak and identify the vehicle of transmission in order to implement control measures to prevent further outbreaks. As the participants of one of the meetings were $\mathrm{HCWs}$, a secondary objective was to explore the compliance of NIPH recommendations for HCWs in case of gastrointestinal illness.

\section{METHODS}

\section{Epidemiological investigation}

We conducted a retrospective cohort study including all the participants of the two meetings by sending them a standardized web-based questionnaire via email on 13 November 2013. A reminder was sent on 18 November and the data collection was closed on 25 November.

The web-based questionnaire collected information on demographics, symptoms and consumption of food and beverages during the meetings. The menu was obtained from the conference centre kitchen. Exposure to food items and beverages was categorized as follows: 'ate/drank the food item or beverage', 'probably ate/drank it', 'probably did not eat/drink it' and 'did not eat/drink it'.

The following three meals were served: a welcome snack buffet, lunch and an afternoon snack buffet. In the welcome snack buffet whole fresh fruit (not sliced), muesli, cereals, yoghurt and nuts were offered. The lunch plate served to every participant included cured ham, potato salad, sour cream, sliced chicken fillet, smoked salmon, scrambled eggs, shrimp, cheese, mixed salad and bread. Raspberry mousse was served as dessert during lunch. The afternoon snack buffet contained sliced fresh fruit, nuts, dried fruits and a carrot cake. Beverages offered during the meetings were coffee, tea, carbonated beverages, yoghurt drink, and tap water from a dispenser.

In order to explore the compliance of NIPH recommendations for healthcare in case of gastrointestinal illness, we asked participants whether they were HCWs and if they had direct contact with patients, and collected information on absence from work related to symptom duration.

\section{Case definition}

We defined a case as diarrhoea and/or vomiting in a participant of either of the two meetings who became ill from noon on 4 November until midnight on 7 November 2013, without household contacts with similar symptoms during the week before onset of symptoms.

Cases for which a date of onset was not given were excluded from the analysis.

\section{Exposure definition}

For analysis purposes 'exposed' was defined as those who answered 'ate/drank the item'. Those who answered 'probably ate/drunk it', 'probably did not eat/drink it' and 'did not eat/drink it' were defined as 'unexposed'.

\section{Statistical analysis}

We performed a descriptive and univariate analysis by calculating attack rates (AR) in exposed and unexposed individuals, risk ratios (RR) with $95 \%$ confidence intervals (CI), and percentage of cases exposed to every food item and beverage. We performed a multivariable 
analysis to identify exposures significantly associated with illness and to control for possible confounders and effect modifiers. In the multivariable analysis we calculated adjusted risk ratios (aRR) in a forward binomial regression including exposures with a $P$ value lower than 0.25 in the univariable analysis. We performed sensitivity analyses to ensure the robustness of our results, relocating the 'probably exposed/unexposed'. In the first sensitivity analysis we recoded 'probably exposed/unexposed' to 'missing', and in the second, 'probably exposed' was recoded to 'exposed' and 'probably unexposed' was recoded to 'unexposed'. Data analysis was performed with Excel 2010 (Microsoft Corp., USA) and Stata v. 12 (StataCorp., USA).

\section{Microbiological investigation}

In a separate email, we encouraged all participants to provide a stool sample if they had developed gastrointestinal symptoms after the meeting. All faecal samples were investigated for Salmonella, Shigella, Campylobacter, and Yersinia by culture and biochemical identification according to standard clinical microbiological methods. Due to the characteristics of the outbreak samples were tested for norovirus by polymerase chain reaction (PCR). Norovirus-positive samples were sent to the Department of Virology at NIPH where the genotyping was done by sequencing of the PCR product ( $\sim 350$ bp product) [8].

\section{Environmental investigation}

NFSA carried out a routine environmental inspection of the kitchen: sampling food leftovers, interviewing the kitchen staff and a performing a traceback of the suspected food items.

\section{RESULTS}

\section{Descriptive epidemiology}

The questionnaire was sent to all 168 people who had attended the two meetings. In total, $147(88 \%)$ responded, of whom $124(84 \%)$ were women and $109(74 \%)$ were in the $40-59$ years age group. Most of the respondents lived in Oslo $(58 \%)$ and the neighbouring municipality Bærum (9\%) with the remainder from 24 other municipalities.

Of the 147 respondents, 85 people (58\%) reported to have become ill, while nine were unsure. A total of 78 $(53 \%)$ developed diarrhoea and/or vomiting.
Table 1. Clinical details of cases $(n=74)$. Norovirus outbreak, Oslo, November 2013

\begin{tabular}{lcc}
\hline \hline Clinical details & $n$ & $\%$ \\
\hline Symptoms & & \\
$\quad$ Nausea & 58 & 78 \\
Vomiting & 51 & 69 \\
Diarrhoea & 50 & 68 \\
Feeling unwell & 47 & 64 \\
Headache & 40 & 54 \\
Abdominal pain & 38 & 51 \\
Muscular pain & 31 & 42 \\
Fever & 23 & 31 \\
Duration of illness & & \\
$\quad<6$ h & 0 & 0 \\
6-12 h & 3 & 10 \\
13-24 h & 25 & 81 \\
22 days* & 3 & 10 \\
GP visit & 2 & 3 \\
Norovirus in stool sample & $5 \dagger$ & 100 \\
Hospitalization & 0 & 0 \\
\hline \hline
\end{tabular}

* Three people reported still being sick at the time they answered the questionnaire 10 days after the meeting. $\dagger$ Only five cases provided a stool sample.

Four cases were excluded from further analysis due to the following reasons: in one case the patient's symptoms developed before 12:00 hours on 4 November; in one case the date of onset was not given; and two casepatients had been in contact with sick household members in the 7 days before onset of symptoms.

In total, 74 cases met the case definition, resulting in an overall attack rate of 50\% (74/147). Sex, age and geographical distribution were similar in cases and non-cases. Nausea and vomiting were the most frequently reported symptoms (Table 1). The date and time of onset for the cases are shown in Figure 1. Most of the cases developed symptoms $24-48 \mathrm{~h}$ after the meeting. The frequency of responses to different exposure categories are shown in Table 2.

\section{Analytical epidemiology}

The association between illness and exposure to food and beverage items calculated in the univariable analysis is shown in Table 3. People who had eaten raspberry mousse (RR 3.4, 95\% CI 1.4-8.4), sliced fresh fruit (RR 1.8, 95\% CI 1.2-2.6) and carrot cake (RR 1.6, $95 \%$ CI $1 \cdot 1-2 \cdot 3$ ) had a significantly higher risk of illness.

Of the four items remaining in the final multivariable model, raspberry mousse (aRR 3.4, 95\% CI 1.4-8.2) and sliced fresh fruit (aRR 1.9, 95\% CI 1.3- 


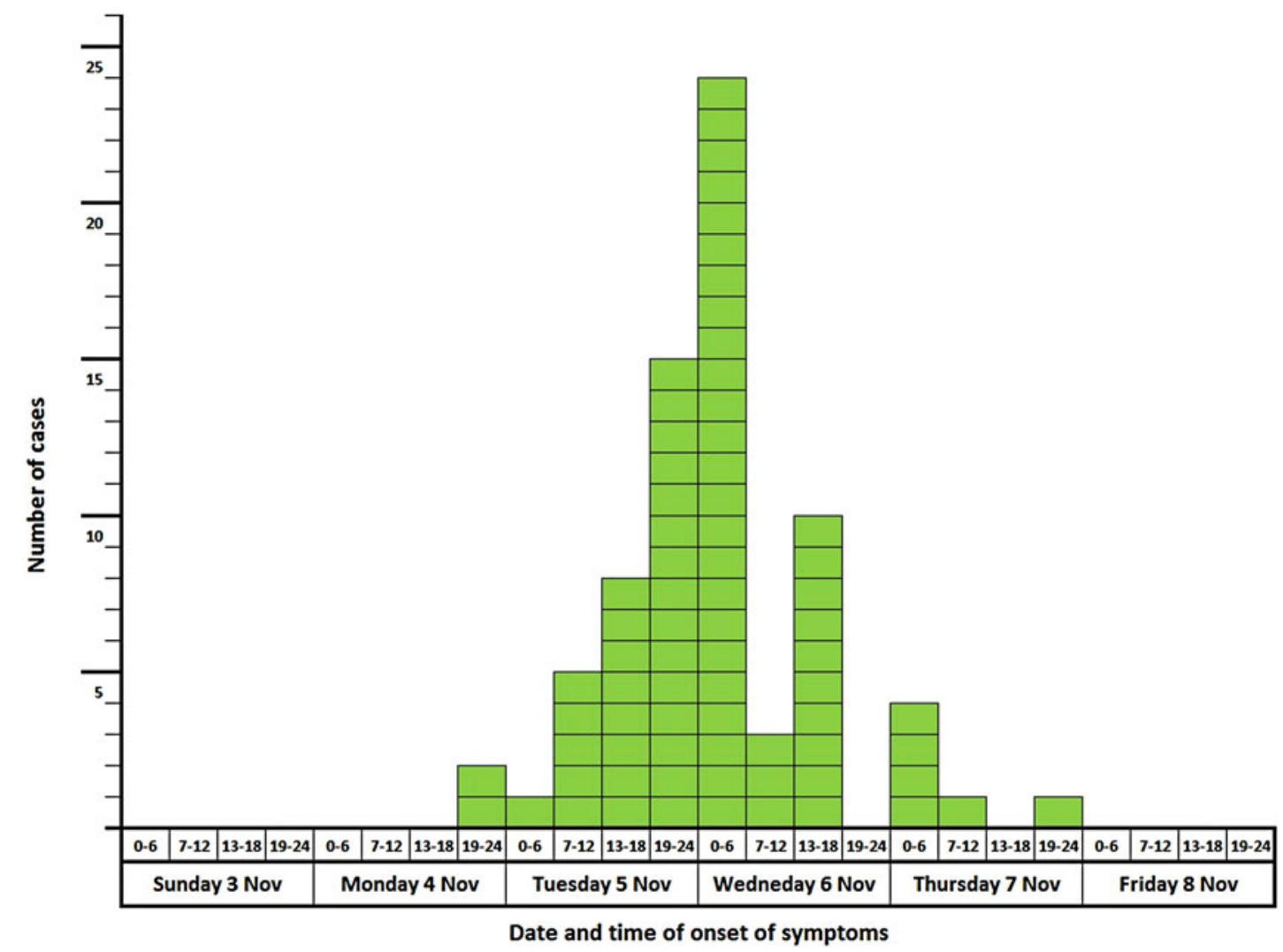

Fig. 1. Date and time of onset of symptoms in 6-h intervals for cases $(n=74)$. Norovirus outbreak, Oslo, November 2013.

$2 \cdot 8)$ were the only two exposures significantly associated with a higher risk of illness (Table 4); whereas whole fresh fruit and cheese were not associated with a higher risk. Of the 74 cases, $70(95 \%)$ ate raspberry mousse and $57(77 \%)$ ate sliced fresh fruit. Of the 57 cases who reported eating sliced fruit, 55 also ate raspberry mousse.

In the first sensitivity analysis, where the categories 'probably exposed' and 'probably unexposed' were recoded as 'missing', the raspberry mousse was the only significant exposure associated with the disease (aRR 2·3, 95\% CI 1·2-7.2). In the second sensitivity analysis, where 'probably exposed' was recoded to 'exposed' and 'probably unexposed' was recoded to 'unexposed', none of the exposures were significant in the multivariable analysis but the raspberry mousse had the smallest $P$ value (aRR $2 \cdot 4,95 \%$ CI $0 \cdot 97-6 \cdot 0$, $P=0 \cdot 060$ ).

A total of $25(17 \%)$ respondents were HCWs, of whom $11(44 \%)$ met the case definition and worked in contact with patients. From these 11, seven provided enough information to explore the compliance of the NIPH recommendations. The mean duration of symptoms was 3 days. Five $(71 \%)$ of those seven did not follow the recommendation of staying at home until $48 \mathrm{~h}$ after the end of symptoms.

\section{Microbiological investigation}

In total, five participants provided stool samples and all were positive for norovirus. Three of the specimens were sent to the Department of Virology of NIPH for genotyping. All three strains belonged to genogroup GI serotype 2 (Southampton).

\section{Environmental investigation}

The NFSA inspected the kitchen on 11 November, a week after the meetings took place. No food items were available for sampling as there were no leftovers from the meetings at this time. None of the kitchen staff reported having had symptoms before and/or on the day of the meeting, but two employees reported becoming ill 2 days after (dishwasher and one waitress). No stool samples were taken from staff members.

All cooked and fresh food items served were prepared in the kitchen of the conference centre. The raspberry mousse and the sliced fresh fruit were not 
Table 2. Frequency of responses to different exposure categories. Norovirus outbreak, Oslo, November 2013

\begin{tabular}{|c|c|c|c|c|c|c|c|c|c|c|}
\hline \multirow[b]{2}{*}{ Exposure } & \multicolumn{2}{|l|}{ Ate } & \multicolumn{2}{|c|}{ Probably ate } & \multicolumn{2}{|c|}{$\begin{array}{l}\text { Probably did } \\
\text { not eat }\end{array}$} & \multicolumn{2}{|c|}{ Did not eat } & \multicolumn{2}{|c|}{ Missing } \\
\hline & $n$ & $\%$ & $n$ & $\%$ & $n$ & $\%$ & $n$ & $\%$ & $n$ & $\%$ \\
\hline Bread & 117 & $79 \cdot 6$ & 2 & $1 \cdot 4$ & 1 & $0 \cdot 7$ & 18 & $12 \cdot 2$ & 9 & 6 \\
\hline Carbonated beverages & 88 & $59 \cdot 9$ & 1 & $0 \cdot 7$ & - & - & 42 & $28 \cdot 6$ & 16 & 11 \\
\hline Carrot cake & 92 & $62 \cdot 6$ & 4 & $2 \cdot 7$ & - & - & 42 & $28 \cdot 6$ & 9 & 6 \\
\hline Cheese & 74 & $50 \cdot 3$ & 20 & $13 \cdot 6$ & 15 & $10 \cdot 2$ & 29 & $19 \cdot 7$ & 9 & 6 \\
\hline Coffee & 74 & $50 \cdot 3$ & 39 & $26 \cdot 5$ & 3 & $2 \cdot 0$ & 25 & $17 \cdot 0$ & 6 & 4 \\
\hline Cured ham & 118 & $80 \cdot 3$ & 7 & $4 \cdot 8$ & 1 & $0 \cdot 7$ & 12 & $8 \cdot 2$ & 9 & 6 \\
\hline Dried fruits & 15 & $10 \cdot 2$ & 7 & $4 \cdot 8$ & 16 & $10 \cdot 9$ & 100 & $68 \cdot 0$ & 9 & 6 \\
\hline Drink yoghurt & 8 & $5 \cdot 4$ & - & - & - & - & 111 & $75 \cdot 5$ & 28 & 19 \\
\hline Mixed salad & 117 & $79 \cdot 6$ & 11 & $7 \cdot 5$ & 3 & $2 \cdot 0$ & 7 & $4 \cdot 8$ & 9 & 6 \\
\hline Muesli/cereals & 26 & $17 \cdot 7$ & - & - & 4 & $2 \cdot 7$ & 108 & $73 \cdot 5$ & 9 & 6 \\
\hline Nuts (1st break) & 42 & $28 \cdot 6$ & 6 & $4 \cdot 1$ & 7 & $4 \cdot 8$ & 83 & $56 \cdot 5$ & 9 & 6 \\
\hline Nuts (2nd break) & 22 & $15 \cdot 0$ & 10 & $6 \cdot 8$ & 16 & $10 \cdot 9$ & 90 & $61 \cdot 2$ & 9 & 6 \\
\hline Potato salad & 114 & $77 \cdot 6$ & 8 & $5 \cdot 4$ & 3 & $2 \cdot 0$ & 13 & $8 \cdot 8$ & 9 & 6 \\
\hline Raspberry mousse & 115 & $78 \cdot 2$ & 2 & $1 \cdot 4$ & - & - & 21 & $14 \cdot 3$ & 9 & 6 \\
\hline Scrambled eggs & 129 & $87 \cdot 8$ & 1 & $0 \cdot 7$ & 2 & $1 \cdot 4$ & 6 & $4 \cdot 1$ & 9 & 6 \\
\hline Shrimp & 124 & $84 \cdot 4$ & 3 & $2 \cdot 0$ & 3 & $2 \cdot 0$ & 8 & $5 \cdot 4$ & 9 & 6 \\
\hline Sliced chicken fillet & 121 & $82 \cdot 3$ & 8 & $5 \cdot 4$ & 2 & $1 \cdot 4$ & 7 & $4 \cdot 8$ & 9 & 6 \\
\hline Sliced fresh fruit & 92 & $62 \cdot 6$ & 10 & $6 \cdot 8$ & 6 & $4 \cdot 1$ & 30 & $20 \cdot 4$ & 9 & 6 \\
\hline Smoked salmon & 122 & $83 \cdot 0$ & 5 & $3 \cdot 4$ & 1 & $0 \cdot 7$ & 10 & $6 \cdot 8$ & 9 & 6 \\
\hline Sour cream & 94 & $64 \cdot 0$ & 14 & $9 \cdot 5$ & 9 & $6 \cdot 1$ & 21 & $14 \cdot 3$ & 9 & 6 \\
\hline Tap water dispenser & 40 & $27 \cdot 2$ & 10 & $6 \cdot 8$ & 1 & $0 \cdot 7$ & 76 & $51 \cdot 7$ & 20 & 14 \\
\hline Tea & 24 & $16 \cdot 3$ & 6 & $4 \cdot 1$ & 1 & $0 \cdot 7$ & 94 & $64 \cdot 0$ & 22 & 15 \\
\hline Whole fresh fruit & 107 & $72 \cdot 8$ & 2 & $1 \cdot 4$ & 2 & $1 \cdot 4$ & 27 & $18 \cdot 4$ & 9 & 6 \\
\hline Yoghurt & 21 & $14 \cdot 3$ & - & - & 3 & $2 \cdot 0$ & 114 & $77 \cdot 6$ & 9 & 6 \\
\hline
\end{tabular}

$n$, Number of respondents.

Table 3. Univariable analysis results. Food and beverage items with $P<0 \cdot 25$. Norovirus outbreak, Oslo, November 2013

\begin{tabular}{|c|c|c|c|c|c|c|c|c|c|c|}
\hline \multirow[b]{2}{*}{ Exposure } & \multicolumn{3}{|c|}{ Exposed } & \multicolumn{3}{|c|}{ Unexposed } & \multirow[b]{2}{*}{ RR } & \multirow[b]{2}{*}{$95 \% \mathrm{CI}$} & \multirow[b]{2}{*}{$P$ value } & \multirow{2}{*}{$\begin{array}{l}\% \text { Cases } \\
\text { exposed }\end{array}$} \\
\hline & Total & Cases & $\mathrm{AR}, \%$ & Total & Cases & $\mathrm{AR}, \%$ & & & & \\
\hline Raspberry mousse & 112 & 70 & 63 & 22 & 4 & 18 & $3 \cdot 44$ & $(1 \cdot 40-8 \cdot 44)$ & $0 \cdot 000$ & 95 \\
\hline Sliced fresh fruit & 88 & 57 & 65 & 46 & 17 & 37 & $1 \cdot 75$ & $(1 \cdot 17-2 \cdot 63)$ & $0 \cdot 002$ & 77 \\
\hline Carrot cake & 89 & 56 & 63 & 45 & 18 & 40 & $1 \cdot 57$ & $(1 \cdot 06-2 \cdot 33)$ & $0 \cdot 012$ & 76 \\
\hline Juice & 21 & 15 & 71 & 98 & 48 & 49 & $1 \cdot 46$ & $(1 \cdot 04-2 \cdot 04)$ & $0 \cdot 061$ & 20 \\
\hline Whole fresh fruit & 103 & 61 & 59 & 31 & 13 & 42 & $1 \cdot 41$ & $(0 \cdot 91-2 \cdot 20)$ & $0 \cdot 090$ & 82 \\
\hline Cheese & 72 & 35 & 49 & 62 & 39 & 63 & $0 \cdot 77$ & $(0 \cdot 57-1 \cdot 05)$ & 0.097 & 47 \\
\hline Nuts (2nd break) & 21 & 15 & 71 & 113 & 59 & 52 & $1 \cdot 37$ & $(0 \cdot 99-1 \cdot 89)$ & $0 \cdot 104$ & 20 \\
\hline Water dispenser & 39 & 24 & 62 & 84 & 40 & 48 & $1 \cdot 29$ & $(0 \cdot 92-1 \cdot 81)$ & $0 \cdot 150$ & 32 \\
\hline Carbonated drinks & 84 & 46 & 55 & 43 & 18 & 42 & $1 \cdot 31$ & $(0 \cdot 87-1 \cdot 96)$ & $0 \cdot 169$ & 62 \\
\hline Scrambled eggs & 125 & 71 & 57 & 9 & 3 & 33 & $1 \cdot 70$ & $(0 \cdot 67-4 \cdot 35)$ & $0 \cdot 172$ & 96 \\
\hline Drink yoghurt & 8 & 6 & 75 & 107 & 54 & 51 & $1 \cdot 49$ & $(0 \cdot 96-2 \cdot 31)$ & $0 \cdot 180$ & 8 \\
\hline Chicken fillet & 117 & 67 & 57 & 17 & 7 & 41 & $1 \cdot 39$ & $(0 \cdot 77-2 \cdot 51)$ & $0 \cdot 213$ & 91 \\
\hline Bread & 115 & 66 & 57 & 19 & 8 & 42 & $1 \cdot 36$ & $(0 \cdot 79-2 \cdot 36)$ & $0 \cdot 214$ & 89 \\
\hline
\end{tabular}

$\mathrm{AR}$, Attack rate; RR, risk ratio; $\mathrm{CI}$, confidence interval. 
Table 4. Final multivariable binomial regression model of food and beverage exposures associated with disease. Norovirus outbreak, Oslo, November 2013

\begin{tabular}{|c|c|c|c|c|c|c|}
\hline \multirow[b]{2}{*}{ Exposure } & \multirow[b]{2}{*}{ Total } & \multicolumn{2}{|c|}{ Exposed } & \multirow[b]{2}{*}{ aRR } & \multirow[b]{2}{*}{$95 \% \mathrm{CI}$} & \multirow[b]{2}{*}{$\%$ cases expose } \\
\hline & & Cases & $\mathrm{AR}, \%$ & & & \\
\hline Raspberry mousse & 112 & 70 & 63 & $3 \cdot 36$ & $1 \cdot 38-8 \cdot 22$ & 95 \\
\hline Sliced fresh fruit & 88 & 57 & 65 & 1.90 & $1 \cdot 32-2 \cdot 76$ & 77 \\
\hline Whole fresh fruit & 103 & 61 & 59 & $0 \cdot 80$ & $0.71-0.92$ & 81 \\
\hline Cheese & 72 & 35 & 49 & 0.69 & $0.52-0.85$ & 47 \\
\hline
\end{tabular}

$\mathrm{AR}$, Attack rate; aRR, adjusted risk ratio.

prepared by the same person. The fresh fruit, both whole and sliced were of the same kind and purchased from the same distributor. The raspberry mousse was prepared with frozen raspberries, powdered sugar, whipped cream, and gelatin. In the kitchen, the raspberries were puréed in a food processor and pressed through a sieve to remove the seeds of the berries to make a coulis. Part of the coulis was heated just enough in order to melt the gelatin and then mixed with the remaining coulis and cream.

The NFSA traced the raspberries back to a country outside of the European Union. A Rapid Alert System for Food and Feed (RASFF) notification had been issued from Denmark on berries originating from the same country just a few weeks after this outbreak. Norovirus was found in the batch in Denmark, but the 'best-before' date was not identical with the batch in Norway. The batch distributed to Norway was no longer on the market, as most of it had already been barred by the distributor due to a labelling error in late summer. The distributor provided the results of laboratory test they had for the batch, which were negative for Salmonella and Listeria. No virus test was provided.

During their inspection in the kitchen, NFSA found some irregularities regarding hand-washing facilities. The sink in the kitchen had no paper dispenser and was located too low.

\section{DISCUSSION}

The outbreak investigation indicates that consumption of raspberry mousse made of raspberries contaminated with norovirus was the most likely vehicle of the outbreak. The raspberry mousse was eaten by $95 \%$ of the cases and had an attack rate of $63 \%$. Those who ate raspberry mousse had more than three times higher risk of becoming ill than those who did not eat raspberry mousse.
Cases occurred in participants in two separate meetings held in different rooms of the conference centre, served by different staff. Contamination of the food items and/or beverages therefore probably occurred before serving. The main symptoms developed by participants, the incubation period, duration of symptoms and the positive stool samples support the theory that the causative agent was norovirus.

In order to address possible recall bias, when allocating the exposure we ran two sensitivity analyses, re-coding 'probably exposed/unexposed' to different categories. Considering the 'probably exposed/unexposed' as 'missing' reduces the sample size and thus power, while considering the 'probably exposed' as 'exposed' and the 'probably unexposed' as 'unexposed' might lead to a misclassification that (in the worst scenario) could overestimate the risk associated with a food/drink item. However, the results of the two sensitivity analyses supported the raspberry mousse as the main vehicle in this outbreak.

The raspberries used in the mousse were imported frozen and they were not heat-treated before consumption. Due to the lack of a cell culture model for norovirus, surrogates of feline and murine calicivirus have been used for environmental survival studies [9-11], showing that norovirus can survive in frozen berries [11]. In addition, frozen raspberries are a known vehicle of outbreaks of norovirus [12-16]. Norovirus contamination of berries can happen during production if berries are irrigated with contaminated water or during handling of the raspberries when collected and processed [17, 18]. Isolation of norovirus from soft berries is challenging due to methodological challenges and the heterogeneous distribution of norovirus in berries, which may lead to false-negative results even with optimized sampling procedures [19].

Four case-patients did not report eating raspberry mousse. An explanation as to why these four fell ill could be that they had been in contact with other 
food/s cross-contaminated from the raspberries or that they were affected by an alternative transmission route. Even if only two case-patients that ate sliced fresh fruit did not eat raspberry mousse, sliced fresh fruit may have been a second vehicle of transmission. The fresh fruit served in the two breaks was the same. Whereas the people who ate the whole fresh fruit during the first break did not have a higher risk of developing the illness, people who ate the sliced fresh fruit during the second break were almost twice as likely to develop symptoms. The main difference was that in the second break the fruit was sliced and contamination could have occurred while cutting the fruit. However, no kitchen staff reported illness before or during the meeting and the person who prepared the raspberry mousse was not the same as the one who sliced the fresh fruit. Nevertheless, contamination of food items by food handling cannot be ruled out as food handlers could have been asymptomatic carriers [20].

Person-to-person transmission is the most common mode of transmission for norovirus outbreaks [21], and two of the participants attending one of the meetings reported having gastrointestinal symptoms on the day of the meeting. The specific time of onset of the vomiting and diarrhoea was not available. We cannot exclude the possibility that norovirus was introduced by a meeting participant, but this is unlikely to be the main route of transmission as the two meetings took place in separate rooms. Regarding compliance of the NIPH recommendations for HCWs and absence from work, our results indicate that the recommendations to refrain from working for $48 \mathrm{~h}$ after symptoms subside may not be routinely followed. However, the sample of HCWs providing sufficient information is too small to make any conclusions and further studies are warranted.

\section{CONCLUSIONS AND RECOMMENDATIONS}

Based in the findings of this investigation we concluded that the most likely vehicle of transmission in this outbreak was the raspberry mousse.

As imported frozen raspberries are a known vehicle of norovirus, the NFSA recommends boiling imported frozen berries for $1 \mathrm{~min}$ before they are used in dishes that are not heat-treated [22]. In order to avoid contamination of berries, it is important to improve hygiene measures during cultivation and harvest.
As the NFSA inspection uncovered irregularities in kitchen hygiene, contamination of raspberries and sliced fresh fruit by food handlers in the kitchen of the conference centre cannot be ruled out. We recommend reinforcing the education of food handlers in hygienic measures for food preparation, and to provide hand-washing facilities in the kitchen according to the recommendations.

\section{ACKNOWLEDGEMENTS}

The authors thank the participants and organizers of the meetings for their collaboration. We thank Katrine Borgen from the Department of Infectious Disease Epidemiology of the Norwegian Institute of Public Health and Alicia Barrasa from the European Programme for Intervention Epidemiology Training (EPIET) for their contributions to the outbreak investigation and feedback on the manuscript, and Emily MacDonald from the Department of Infectious Disease Epidemiology of the Norwegian institute of Public Health for her feedback on the manuscript.

This research received no specific grant from any funding agency in the public, commercial, or not-forprofit sectors

\section{DECLARATION OF INTEREST}

None.

\section{REFERENCES}

1. Atmar RL. Noroviruses - state of the art. Food and Environmental Virology 2010; 2: 117-126.

2. Zheng DP, et al. Norovirus classification and proposed strain nomenclature. Virology 2006; 346: 312-323.

3. Glass RI, Parashar UD, Estes MK. Norovirus gastroenteritis. New England Journal of Medicine 2009; 361: 1776-1785.

4. Barclay L, et al. Infection control for norovirus. Clinical Microbiology and Infection 2014; 20: 731-740.

5. Ahmed SM, et al. Global prevalence of norovirus in cases of gastroenteritis: a systematic review and meta-analysis. Lancet Infectious Diseases 2014; 14: 725-730.

6. Atmar RL, et al. Norwalk virus shedding after experimental human infection. Emerging Infectious Diseases 2008; 14: 1553-1557.

7. Norwegian Institute of Publich Health. Norovirus magetarminfeksjoner i kommunale helseinstitusjoner (http:// wwwfhino/dokumenter/1600c0cfd7pdf). Accessed 22 December 2014.

8. Vinjé J, Koopmans MP. Molecular detection and epidemiology of small round-structured viruses in 
outbreaks of gastroenteritis in the Netherlands. Journal of Infectious Diseases 1996; 174: 610-615.

9. Cannon JL, et al. Surrogates for the study of norovirus stability and inactivation in the environment: a comparison of murine norovirus and feline calicivirus. Journal of Food Protection 2006; 69: 2761-2765.

10. Lamhoujeb S, et al. Evaluation of the persistence of infectious human noroviruses on food surfaces by using realtime nucleic acid sequence-based amplification. Applied and Environmental Microbiology 2008; 74: 3349-3355.

11. Butot S, Putallaz T, Sanchez G. Effects of sanitation, freezing and frozen storage on enteric viruses in berries and herbs. International Journal of Food Microbiology 2008; 126: 30-35.

12. Sarvikivi E, et al. Multiple norovirus outbreaks linked to imported frozen raspberries. Epidemiology and Infection 2012; 140: 260-267.

13. Hjertqvist M, et al. Four outbreaks of norovirus gastroenteritis after consuming raspberries, Sweden, JuneAugust 2006. Eurosurveillance 2006; 11: pii $=3038$.

14. Falkenhorst $\mathbf{G}$, et al. Imported frozen raspberries cause a series of norovirus outbreaks in Denmark, 2005. Eurosurveillance 2005; 10: pii $=2795$.

15. Le Guyader FS, et al. Detection of noroviruses in raspberries associated with a gastroenteritis outbreak. International Journal of Food Microbiology 2004; 97: 179-186.

16. Cotterelle B, et al. Outbreak of norovirus infection associated with the consumption of frozen raspberries,
France, March 2005. Eurosurveillance 2005; 10: pii = 2690.

17. Heaton JC, Jones K. Microbial contamination of fruit and vegetables and the behaviour of enteropathogens in the phyllosphere: a review. Journal of Applied Microbiology 2008; 104: 613-626.

18. Grondahl-Rosado R, et al. A one year study on the concentrations of norovirus and enteric adenoviruses in wastewater and a surface drinking water source in Norway. Food and Environmental Virology 2014; 6: 232-245.

19. Mäde D, et al. Detection and typing of norovirus from frozen strawberries involved in a large-scale gastroenteritis outbreak in Germany. Food and Environmental Virology 2013; 5: 162-168.

20. Franck KT, et al. Sources of calicivirus contamination in foodborne outbreaks in denmark, 2005-2011 - the role of the asymptomatic food handler. Journal of Infectious Diseases 2015; 211: 563-570.

21. Blanton LH, et al. Molecular and epidemiologic trends of caliciviruses associated with outbreaks of acute gastroenteritis in the United States, 2000-2004. Journal of Infectious Diseases 2006; 193: 413-21.

22. Norwegian Food Safety Authority. Information about food and health from public authorities (http:// wwwmatportalenno/matvaregrupper/tema/gronnsaker_ frukt_og_bar/skyll_eller_kok_frukt_baer_og_gronnsaker). Accessed 22 December 2014. 